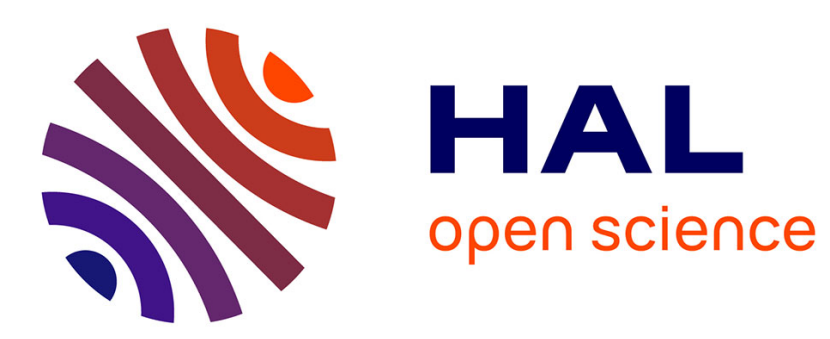

\title{
Genetic algorithms for condition-based maintenance optimization under uncertainty
}

M. Compare, F. Martini, Enrico Zio

\section{To cite this version:}

M. Compare, F. Martini, Enrico Zio. Genetic algorithms for condition-based maintenance optimization under uncertainty. European Journal of Operational Research, 2015, 244 (2), pp.611-623. 10.1016/j.ejor.2015.01.057 . hal-01269867

\section{HAL Id: hal-01269867 \\ https://hal.science/hal-01269867}

Submitted on 5 Feb 2016

HAL is a multi-disciplinary open access archive for the deposit and dissemination of scientific research documents, whether they are published or not. The documents may come from teaching and research institutions in France or abroad, or from public or private research centers.
L'archive ouverte pluridisciplinaire HAL, est destinée au dépôt et à la diffusion de documents scientifiques de niveau recherche, publiés ou non, émanant des établissements d'enseignement et de recherche français ou étrangers, des laboratoires publics ou privés. 


\title{
Genetic Algorithms for Condition-Based Maintenance Optimization under uncertainty
}

\author{
M.Compare ${ }^{1}$, F. Martini ${ }^{2}$, E. Zio ${ }^{1,2,3}$ \\ ${ }^{1}$ Aramis s.r.l., Milano, Italy michele.compare@aramis3d.com \\ ${ }^{2}$ Dipartimento di Energia, Politecnico di Milano, Italy \\ ${ }^{3}$ Chair on Systems Science and Energetic Challenge, European Foundation for New Energy-Electricité de \\ France, Ecole Centrale Paris and Supelec, Paris, France \\ enrico.zio@ecp.fr \\ enrico.zio@polimi.it
}

\begin{abstract}
This paper proposes and compares different techniques for maintenance optimization based on Genetic Algorithms (GA), when the parameters of the maintenance model are affected by uncertainty and the fitness values are represented by Cumulative Distribution Functions (CDFs). The main issues addressed to tackle this problem are the development of a method to rank the uncertain fitness values, and the definition of a novel Pareto dominance concept. The GA-based methods are applied to a practical case study concerning the setting of a condition-based maintenance policy on the degrading nozzles of a gas turbine operated in an energy production plant.
\end{abstract}

\section{Introduction}

Multi-state degradation modelling has recently received considerable attention in the domain of reliability and maintenance engineering [2], [7], [36], [45], [46], as it pragmatically allows setting advanced maintenance paradigms such as Condition-Based Maintenance (CBM) and Predictive Maintenance [62], [63]. In practice, the parameters governing the stochastic transitions among the states of these models are first estimated, based on the available data; then, the degradation model is embedded into the maintenance model to estimate the performance indicators of interest (e.g., unavailability [34], [47], profitability [1], quality in production [11], total costs, risk [19], etc.): this model is at the basis of the optimization algorithm that identifies the set of optimal maintenance settings among which the decision maker selects the preferred solution (e.g., [28], [35]).

On the other side, the correct processing of the uncertainty in the maintenance models is emerging to be a crucial issue for the proper decision on the preferred maintenance solution to apply in practice without 'surprises'. This importance is witnessed by the large amount of literature produced on this topic (e.g., [3], [5], [6], [9], [21], [45], [46]). In other words, the maintenance models have to take into account the uncertainties affecting their parameters, which usually come from limited evidence available in the field. Such epistemic uncertainty (i.e., due to insufficient knowledge) needs to be propagated together with the aleatory uncertainty (i.e., due to the inherent stochastic nature of the 
degradation and failure phenomena), through the maintenance model, onto the considered (maintenance) performance indicators.

Several theoretical frameworks and computational methods have been developed to incorporate imprecise parameters into Markov or semi-Markov multi-state degradation models, when the imprecision is represented by interval probabilities [15], [29], [50], [55], fuzzy stets [20], possibility distributions [3], [6] and probability assignments [5], [9]. However, the problem (undoubtedly difficult [17]) of how to optimize maintenance in the setting where the epistemic and aleatory uncertainties in the embedded degradation model lead to uncertain objective functions (e.g., unavailability, cost, etc.), has not received the necessary attention. In fact, as pointed out in [17] and in [49], few approaches have been propounded in the literature to effectively tackle such multiobjective optimization problems in the presence of uncertain objective functions. These works consider different frameworks for uncertainty representation: probability distributions in [17], [23], [39], [49], [52], [57] fuzzy sets in [31] and [56], and plausibility and belief functions in [14], [33].

In this context, the Authors have proposed methodologies in the framework of Possibility Theory (PT, [3], [6]) and Dempster-Shafer Theory of Evidence (DSTE, [5], [17]) to represent and propagate the uncertainty in multi-state degradation maintenance models, and also to optimize the CBM policy based on the model outputs, which are pairs of plausibility and belief functions. Now, the aim of the present work is to propose an extension of Multi-Objective Genetic Algorithms (MOGA [14], [27], [35], [38], [43]) to tackle the maintenance optimization issue when the epistemic uncertainty in the degradation model is represented in the probability theory framework. Namely, the parameters of the stochastic model of the degradation mechanisms are supposed to be Maximum Likelihood (ML)estimated and the uncertainties in these estimations are represented by probability distributions [4], [25], [48]. A double-loop Monte-Carlo approach [61] is used to propagate the uncertainties from the model parameters onto the considered performance indicators (i.e., the objective functions of the optimization, which represent fitness values of the solutions), which turn out to be probability distributions.

The development of a technique to rank the uncertain fitness values and the generalization of the Pareto dominance concept are the two fundamental issues to address in order to extend the application of MOGA to the case of uncertain objective functions, being the evolutionary schemes wellestablished frameworks in which these innovations have to be embedded. In this work, we propose the pairwise ranking technique propounded in [10] and [14], and a Pareto dominance concept based on the ranks of the solutions. These concepts are combined with the NSGA-II elitist technique ([16]), which relies on the Pareto dominance to effectively divide the evolving populations into non- 
dominated fronts of different ranks, thus allowing for a significant reduction in the computational times.

Finally, the results provided by this technique are compared to those of the CVaR approach [41], [53], which condenses the uncertainties in the objective functions into crisp values, representative of the associated risk. The comparison of these techniques and of their performances constitutes an additional original contribution of the paper.

The remainder of the paper is organized as follows. Section 2 describes the ranking criterion and the Pareto dominance definition; Section 3 takes a glance at the GA advancements considered in this work (named extended NSGA-II, hybrid NSGA-II and CVaR measure). A practical case study is introduced in Section 4, which concerns the optimization of a CBM policy on the nozzle system of gas turbines. The results of the application of the proposed methodologies to the case study are shown and discussed in Section 5. Concluding remarks are given in Section 6.

\section{Sorting Method}

In the uncertainty setting considered in this work, finding the optimal set that minimizes the objective functions (e.g., unavailability and cost) requires developing a method to establish a relation order among two probability distributions. To this aim, we consider the algorithm proposed in [10], which is briefly recalled in this Section.

Let us consider two generic random variables $A$ and $B$. To establish which is the largest, we consider the random variable $\Delta_{A B}=A-B$. Then, the probability that $A$ is larger than $B$, referred to as "exceedance measure", is given by $r_{A B}=1-F_{\Delta_{\mathrm{AB}}}(0)$, where $F_{\Delta_{\mathrm{AB}}}$ is the Cumulative Distribution Function (CDF) of $\Delta_{A B}$.

The relationship between $A$ and $B$ is obtained by comparing $r_{A B}$ to a threshold range $\left[T_{l}, 1-T_{l}\right]$, symmetric around 0.5 , and considering the following criteria:

- If $r_{A B} \geq 1-T_{l}$, then $A$ is larger than $B$.

- If $r_{A B} \leq T_{l}$, then $B$ is larger than $A$.

- If $T_{l} \leq r_{A B} \leq 1-T_{l}$, then $A$ is equal to $B$.

In practice, $B$ is the largest among the two if the decision-maker judges the probability of $B$ being larger than $A$ 'large' enough (e.g., 0.7). A different point of view may be that $A$ and $B$ are similar as long as $r_{A B}$ belongs to $\left[T_{l}, 1-T_{l}\right]$. This way, the exceedance measure becomes a similarity measure. 
This method is similar to that proposed in [18], which also relies on the pairwise comparisons of the probability distributions. However, the solution proposed to solve multi-criteria decision problems is completely different from that proposed in this work.

For example, Figure 1 shows the Probability Density Functions (PDFs) of two variables $A$ and $B$, (left) and the corresponding CDFs (center). The results of the application of the sorting algorithm are shown in Figure 1 (right): the CDF of $\Delta_{A B}$ indicates that the exceedance measure $r_{A B}=P(A>B)=$ $1-F_{A B}(0)=0.8$; this allows concluding that $A>B$.

Figure 1 also highlights the drawback of the procedures to sort the probability distributions, which rely on points summarizing the distributions (e.g., the approach proposed in [12]). Namely, the expected value of $A$, (i.e, the middle point of the distribution) $E[A]$, is larger than that of $B, E[B]$. On the other hand, the $90^{\text {th }}$ percentile of $A$ is smaller than the $90^{\text {th }}$ percentile of $B$. This entails that if one were to perform the ranking based on the $90^{\text {th }}$ percentile values the conclusion would be that $B$ is larger than $A$, contrarily to what would be happen if the ranking were based on the expected values. Figure 1 allows us also underlining the difference between the proposed ranking method and the classical definition of stochastic ordering (the so called 'usual' stochastic order, e.g., [30], [54]): $B$ is smaller than $A$ if $F_{\mathrm{A}}(\mathrm{x}) \leq F_{\mathrm{B}}(\mathrm{x}), \forall \mathrm{x} \in \mathbb{R}$. Namely, the CDFs plotted in Figure 1 (center) intersect, and the condition for having one curve dominating the other is not fulfilled. Thus, $A$ and $B$ need to be considered as 'equal', although $P(A>B)=0.8$. On the contrary, Figure 2 shows the situation where $A \approx N(\mu=2.1, \sigma=1)$ is larger than $B \approx N(\mu=2, \sigma=1)$ in the usual stochastic order, although the probability that $A>B$ is very poor $(A-B \approx N(\mu=0.1, \sigma=\sqrt[2]{2})$, and $P(A>B)=$ $0.53)$.

The same considerations hold when the third degree stochastic dominance is applied [58]: one can establish an order relation between $A$ and $B$ even if the evidence that $A$ is larger than $B$ is very small. From these considerations, it seems fair to say that the ranking methodology proposed in [10] is more capable of capturing the information contained in the CDFs.
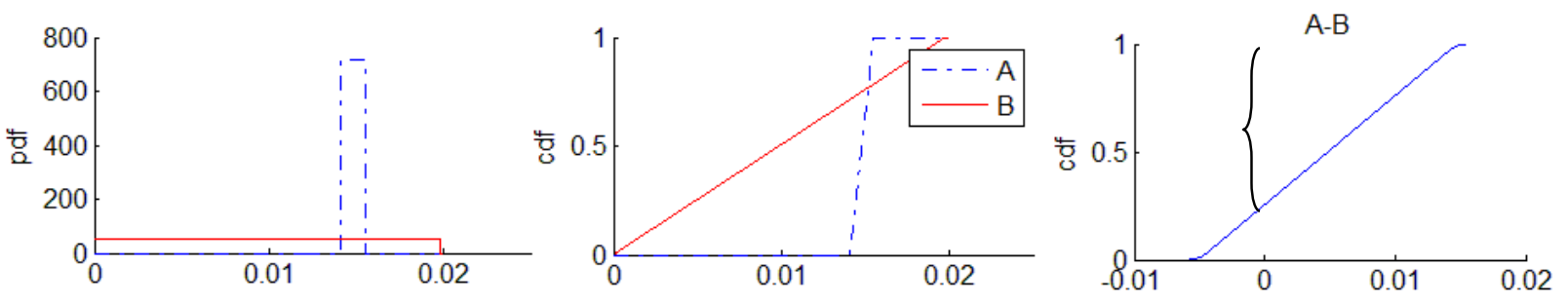

Figure 1: two PDFs and CDFs comparison

Finally notice that a ranking technique has been proposed in [49] in support to GA, which establishes the order relation based on the comparison of the solutions with an ideal, pre-fixed Dirac delta 
distribution. It is worth noticing that when the reference Dirac delta is positioned at zero, then the method proposed in [49] reduces to sorting the distributions based on their mean values. Then, also in this case the CDF sorting is based on points summarizing the CDFs, thus losing the information they encode.
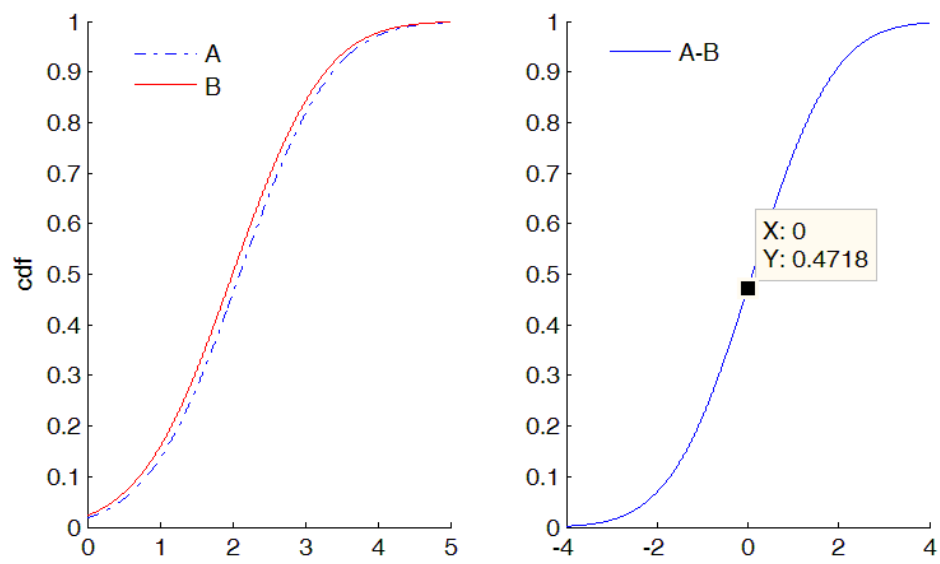

Figure 2: two PDFs and CDFs comparison

The authors in [10] also pointed out that there may be cases in which the pairwise comparisons of three generic random variables $A, B$ and $C$ lead to $A>B$ and $B>C$, but $C>A$. This is a 'contradictory' ranking, as the transitive property does not hold. However, it has been proven in [8] that by setting $T_{l}$ smaller than $1 / 3$, such contradictory ranking is avoided and, at most, it can happen that $A>B, B>C$, and $C=A$. In this case, the three uncertain variables are considered equivalent.

Notice that the loss of the transitive property for similarity measures is a well-known issue, whose raising dates back to the 50's of the last century [42]: Poincaré emphasized that in the observable physical continuum, "equal" means "indistinguishable," and $\mathrm{A}=\mathrm{B}$ and $\mathrm{B}=\mathrm{C}$ do not imply $\mathrm{A}=\mathrm{C}$ (i.e., physical equality is a non-transitive relation). These considerations have been formally modelled in a number of works (e.g., [37], [22]). Nonetheless, the problem of the 'contradictory' ranking did not emerge in the works of the literature that propose extensions of GA to treat noisy fitness values (e.g., [17], [23]).

\subsection{Pareto Dominance}

When sorting of elements concerns multiple attributes, the concept of Pareto dominance is introduced. Assume that there are $Q$ objectives $\Theta^{1}, \ldots, \Theta^{Q}$ to be minimized; then, a feasible solution, say $X_{S}$, is dominated by another feasible solution, say $X_{j}$, if for all $q=1, \ldots, Q$ the corresponding values of the objectives, $\Theta_{s}^{q}$ and $\Theta_{j}^{q}$, are such that [27], [38]:

1. $\Theta_{s}^{q} \leq \Theta_{j}^{q}, \forall q=1, \ldots, Q$, and

2. $\Theta_{s}^{q}<\Theta_{j}^{q}$ at least for one $q=1, \ldots, Q$. 
If $X_{s}$ dominates $X_{j}$, then this is indicated by $X_{s}>X_{j}$.

A solution is said to be Pareto optimal if it is not dominated by any other solution in the solution space. This means that a Pareto optimal solution cannot be improved with respect to any objective without worsening at least one other objective. The set of all feasible non-dominated solutions is referred to as the Pareto optimal set; the objective function values corresponding to a given Pareto optimal set form the Pareto front in the objective space.

When the exceedance-based sorting method described above is used to establish if $\Theta_{s}^{q} \leq \Theta_{j}^{q}$, for any $q=1, \ldots, Q$, the given definition of Pareto dominance needs to be modified [14]. To prove this, for the sake of simplicity we can refer to the case of $Q=2$. On one side, one may have that the exceedance measure $r_{s j}^{1}=P\left(\Theta_{s}^{1}>\Theta_{j}^{1}\right) \geq 1-T_{l}$ and $T_{l} \leq r_{s j}^{2}=P\left(\Theta_{s}^{2}>\Theta_{j}^{2}\right) \leq 1-T_{l}$, which leads to conclude that $X_{s}>X_{j}$; on the other side, one may have also that $r_{s j}^{1} \geq 1-T_{l}, r_{j g}^{1} \geq 1-T_{l}$ and $1-T_{l} \geq r_{s g}^{1} \geq T_{l}$ (i.e., $X_{s}, X_{j}$ and $X_{g}$ are of the same rank with respect to the second objective): this means that $\Theta_{s}^{1}$ is equivalent to $\Theta_{j}^{1}$, with respect to objective 1 , and thus $X_{s}$ does not dominate $X_{j}$.

To overcome this issue, the sorting algorithm shown in [8] is first applied to every objective $q=$ $1, \ldots, Q$. This algorithm exploits the ranking criterion described above and assigns the same ranking position to all the solutions that are equivalent with respect to objective $q$. For example, if we have $H=4$ solutions and the second and third solutions are equivalent with respect to objective $q$, then the final ranking is $1,2,2,4$.

Then, the following definition of Pareto dominance is introduced to identify the Pareto front:

$$
X_{s}>X_{j} \text { if } \rho_{s}^{q} \leq \rho_{j}^{q} \text { for all } q=1, \ldots, Q \text { and } \rho_{s}^{q}<\rho_{j}^{q} \text { for at least one } q=1, \ldots, Q
$$

where $\rho_{s}^{q}$ and $\rho_{j}^{q}$ are the ranking positions of the solutions $X_{s}$ and $X_{j}$ with reference to objective $q$, respectively.

Finally, notice that the approach proposed in [49] to implement the NSGA-II algorithm also relies on the ranking positions of the solutions in a population. However, the definition of the rank in [49] is different from that given in this work. Rather, it resembles the ranking method proposed in [44], which applies the Monte Carlo sampling method to estimate, for every solution $\mathrm{X}_{\mathrm{h}}$, the probabilities of occupying the $\mathrm{H}$ positions in the ranking. The final ranking of the solution $\mathrm{X}_{\mathrm{h}}$ is the average value of its ranking positions.

\section{Multi Objective Genetic Algorithm (MOGA) for Optimization}


GA is the most commonly known evolutionary algorithm for optimization, which uses techniques inspired by natural evolution to allow a population of solutions (also called, individuals), candidates to solve the (multiobjective) optimization problem, to evolve, i.e., move toward the best solution.

The evolution usually starts from a population of randomly generated individuals, which change at each iteration, called a generation. In each generation, the fitness (the values of the objective functions) of every individual in the population is evaluated, and the most fitting individuals (those with largest or smallest objective functions values, depending on whether the aim is maximization or minimization, respectively) are selected. Each individual is modified by mating and a new, more evolved, generation of candidate solutions is formed.

Commonly, the algorithm terminates when either a pre-set maximum number of generations has been produced, or a satisfactory fitness level has been reached in the population.

In this Section, the general procedure of the MOGA developed in our work is given as follows [27], [38] (see Figure 3):

Step 1. (Initialization)

Set $t=1$. Randomly generate $H$ solutions to form the first population $X^{t=1}=\left\{X_{1}, \ldots, X_{H}\right\}$.

In the specific case of the maintenance optimization problem, a solution is generally a vector of decision variables such as the time interval between two successive inspections, the type of maintenance action to be performed, etc.

\section{Step 2. (Fitness Evaluation)}

Evaluate the fitnesses of the solutions in $X^{t}$ for every objective $\Theta^{1}, \ldots, \Theta^{Q}$, and assign the corresponding rank value by applying the sorting algorithm shown in [8]. In the uncertainty setting considered in this work, performing this step requires running the double loop Monte Carlo (MC) approach for propagating the uncertainty related to every solution $X_{h} \in X^{t}, h=1, \ldots, H$. That is, the following procedure is implemented:

- An external loop samples the values of the parameters of the multistate model from a normal distribution centred in the MLE values, with the estimated covariance matrix [25] [48].

- For every set of sampled parameters, an internal MC loop propagates the aleatory uncertainty into the maintenance model, and estimates the values of all the objectives $\Theta^{1}, \ldots, \Theta^{Q}$. These values are collected to infer the CDFs of the fitnesses at Step 3.

- At the end of the double loop procedure, all the collected estimations are used to infer the empirical CDFs of the objective functions $\Theta^{1}, \ldots, \Theta^{Q}$. 
- The algorithm proposed in [8] is applied to get the rank position $\rho_{h}^{q}$ of the solution $X_{h} \in X^{t}$, with respect to the objective $q, \forall h=1, \ldots, H, \forall q=1, \ldots, Q$. Finally, the definition of Pareto dominance given in Section 2 is used to identify the set $P^{t}$ of non-dominated solutions in the population $X^{t}$.

Step 3. (Breeding).

Generate an offspring population $W^{t}=\left\{W_{1}, \ldots, W_{H}\right\}$ as follows:

I. $\quad$ Selection

Choose two solutions $X_{S}$ and $X_{l}$ from $X^{t}$. This choice is usually based on the ranking values, and heavily influences the performance of the GA, which is typically evaluated in terms of effectiveness and efficiency. In this work, we apply the dominance depth criteria characterizing the NSGA-II [16], which is one of the most efficient evolutionary algorithms to solve multi-objective optimization problems [41]. The main characteristic of NSGA-II is the Fast Non Dominated Sorting (FNDS) function, used for the selection phase, which allows grouping a population into different non-domination levels. That is, all non-dominated individuals in the current population are identified. These solutions are assigned the rank 1. Then, they are virtually removed from the population and the next set of non-dominated individuals are identified and assigned rank 2 . This process continues until every solution in the population has been ranked. The selection procedure is then based on this ranking: individuals are randomly selected from the same rank class (Figure 3). The rationale of this choice is that every individual belonging to the same rank class can be considered equivalent to any other of the class, i.e., it has the same probability of the others to be selected as a parent and survive the replacement.

II. Crossover

Using a crossover operator, generate offsprings and add them to $W^{t}$.

III. Mutation

Mutate each solution $\left\{W_{1}, \ldots, W_{H}\right\}$ with a predefined mutation rate. This means that the genomes of the individuals are randomly changed, to favour the genetic diversity.

IV. Fitness assignment

For every solution $W_{h}$ in $W^{t}=\left\{W_{1}, \ldots, W_{H}\right\}$, estimate the values of the objective functions $\Theta^{1}, \ldots, \Theta^{Q}$ and the rankings of the solutions with respect to the objectives, by applying to $W^{t}$ the procedure described at Step 2.

\section{V. $\quad$ Replacement}


An archive of vectors is introduced, which contains the non-dominated solutions and the corresponding fitness values. This archive represents the current Pareto optimal set, which is dynamically updated at the end of each generation. That is, the solutions in $P^{t}$ unite those already stored in the archive $A^{t}$, where $A^{1}=\emptyset$. This means that $A^{t+1}=A^{t} \cup P^{t}$. Then, the solutions in $A^{t+1}$ are again sorted with respect to every objective to get the dominance relationships. The following archival rules are implemented:

- The dominated members are removed from $A^{t+1}$;

- otherwise:

- if the archive is not full, $A^{t+1}$ is stored as it is, and it will be used at the next iteration.

- if the archive is full, the solutions most similar to solutions already existing in the archive are removed from $A^{t+1}$. In this respect, an appropriate concept of distance is that of the Euclidean distance based on the values of the fitness of the chromosomes normalized to the respective mean values in the archive.

\section{Step 5. (Stopping criterion)}

If the stopping criterion is satisfied, terminate the search and return to the current population, else, set $t=t+1$ and go back to Step 3. In this respect, notice that there are many stopping criteria (e.g., [38]). In this work, the algorithm terminates when the number of simulation reaches a pre-fixed threshold.

Notice that along with convergence to the Pareto-optimal set, it is also desired that an evolutionary algorithm maintains a good spread of solutions in the obtained set of solutions. In NSGA-II, this is achieved through the crowding-distance computation [16], which guides the selection process at the various stages of the algorithm toward a uniformly spread-out Pareto optimal front. The crowdingdistance computation requires sorting the population according to each objective function value in ascending order of magnitude. This is done by applying the sorting algorithm summarized in Section 2 .

A final consideration concerns the loss of the transitive property introduced by the sorting methods that rely on similarity measures: the problem of the 'contradictory' ranking did not emerge in the works of the literature that propose extensions of GA to treat noisy fitness values (e.g., [17], [23]). This situation is due to the fact that assigning different ranking positions to solutions with equal fitness values does not significantly affect the effectiveness of the Single Objective GA search of the optimal solution; rather, the GA efficiency (i.e., speed of convergence) may be weakened. For example, assume that the fit-fit approach is considered in the reproduction phase [38], and that there 
are $\mathrm{n}$ solutions with equal fitness values. When we sort them in the corresponding ranking positions $\mathrm{i}, \mathrm{i}+1, \ldots, \mathrm{i}+\mathrm{n}-1$, each solution occupies a rank, which depends on the sorting algorithm, or even on the particular run of the algorithm (e.g., the Quicksort algorithm may randomly choose the pivot element [26]). Now, the fit-fit algorithm selects and mates members of these $\mathrm{n}$ solutions. This is a locally hybrid reproduction approach, which is between the fit-fit and random selection approaches, in the sense that, for those $n$ positions, and at most the two neighborhoods in positions $i-1$ and $i+n$, there is a random facet behavior entering the selection of the parents. This may be even beneficial for GA, as it combines the speed of the fit-fit technique with the capability of preserving genetic diversity, typical of the random selection method (see [38] for references). However, the systematic study to assess the impact that such local-hybridization of the selection algorithm has on efficiency and effectiveness is outside the scope of this work.

\subsection{Hybrid NSGA-II and CVaR measure}

In this work, the NSGA-II paradigm is also combined with the $C V a R_{\alpha}$ method [41]. $C V a R_{\alpha}$ is a coherent measure of the risk associated to an uncertain function of interest, which has been broadly used in financial portfolio optimization to either reduce or minimize the probability of incurring in large losses [40].

The definition of the $C V a R_{\alpha}$ risk measure is derived from that of value at risk ( $\left.V a R_{\alpha}\right)$. Namely, let $X$ be a random variable representing the uncertain losses in a given time horizon, and let its CDF be $F_{X}(x)=P(X \leq x)$; then, $\operatorname{VaR}_{\alpha}(X)$ is the $\alpha$-percentile of the random variable $X$. That is, $\operatorname{VaR}_{\alpha}(X)$ represents the smallest value of losses such that the probability of having losses exceeding $\operatorname{VaR}_{\alpha}(X)$ is smaller than $1-\alpha$ (Figure 4$)$.

$V a R_{\alpha}$ is commonly used in many engineering areas involving uncertainties, such as military, nuclear, material, aerospace, finance, etc. [53]. In the case addressed in this work, the losses concern the unavailability and cost. That is, $\operatorname{Var}_{\alpha}^{1}$ and $\operatorname{Var}_{\alpha}^{2}$ are the $\alpha$-percentile of the unavailability and costs, respectively, associated to the CBM policy in a given time horizon $T$. These values have a clear interpretation by the maintenance decision maker: the probability of having losses in availability larger than $\operatorname{Var}_{\alpha}^{1}$ is smaller than $1-\alpha$; the same holds for money losses. 


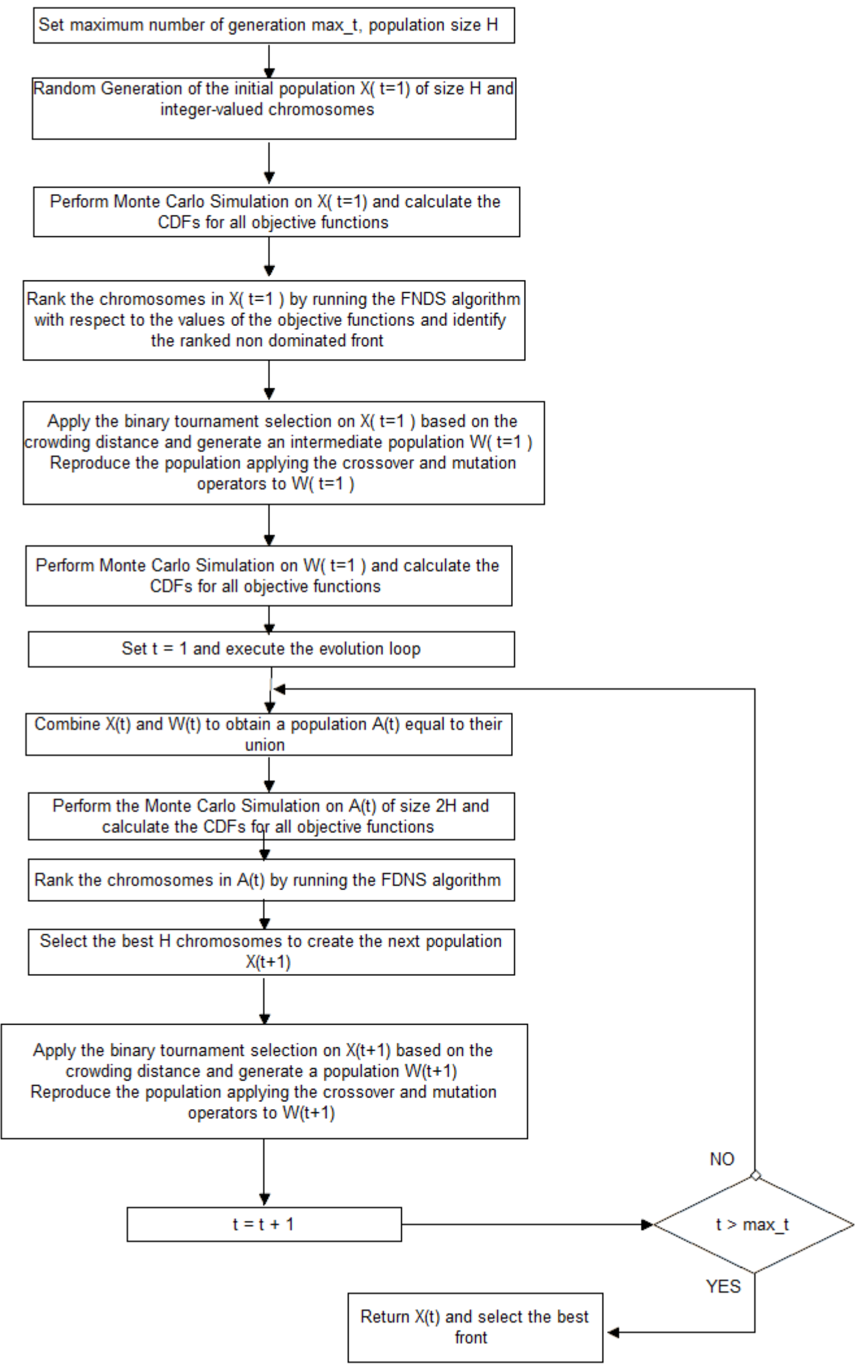

Figure 3: Flow Diagram of NSGA-II algorithm 
The $\operatorname{CVaR}_{\alpha}$ [53] of $X$ with confidence level $\alpha \in(0,1)$ is the mean of the generalized $\alpha$-tail distribution:

$$
\operatorname{CVaR}_{\alpha}(X)=\int_{-\infty}^{+\infty} x d F_{X}^{\alpha}(x)
$$

where

$$
F_{X}^{\alpha}(x)=\left\{\begin{array}{c}
0 \text { when } x<\operatorname{VaR}_{\alpha}(X) \\
\frac{F_{X}(x)-\alpha}{1-\alpha} \text { when } x \geq \operatorname{VaR}_{\alpha}(X)
\end{array}\right.
$$

In turn, we consider the $1-\alpha$-tail $\mathrm{CDF}$, which represents the risk beyond the $\operatorname{VaR}_{\alpha}$. Then, $C V a R_{\alpha}$ represents the mean value of this tail. Figure 4 shows the representation of both $\operatorname{VaR}_{\alpha}$ and $C V a R_{\alpha}$ risk measures.

Also, $C V a R_{\alpha}$ has a clear engineering interpretation. For example, $C \operatorname{Var}_{\alpha}^{1}(U) \leq U^{-}$ensures that the average of $(1-\alpha) \%$ highest losses in availability does not exceed $U^{-}$.

From these definitions, it comes out that the $\operatorname{VaR}_{\alpha}$ and $C V a R_{\alpha}$ risk measures have different meanings and, then, mathematical properties. The problem of the choice between $\operatorname{VaR}_{\alpha}$ and $C V a R_{\alpha}$ has received increasing attention, especially in financial risk management [53]. Anyway, in our case we consider the $C V a R_{\alpha}$ risk measure, because it is more conservative than $V a R_{\alpha}$, which is particularly important when safety is a concern in the application. In particular, following [41], we modify the definition of the objective functions and take a convex combination of the average values of the objectives and their $C V a R_{\alpha}$. That is, we introduce two new objective functions:

$$
\begin{aligned}
& -\Theta^{1}=\beta \times E\left[\Theta^{1}\right]+(1-\beta) \times C \operatorname{Var}_{\alpha}^{1} \\
& -\Theta^{2}=\beta \times E\left[\Theta^{2}\right]+(1-\beta) \times C \operatorname{Var}_{\alpha}^{2}
\end{aligned}
$$

This allows considering different scenarios; that is:

- In case we set $\beta=0$, then we conservatively aim at minimizing the risk of having large unavailability and costs.

- In case of $\beta=1$, the objectives we want to minimize are the mean cost and mean unavailability of the nozzle system.

- In case of $\beta=0.5$, the objective functions are an equal compromise between the corresponding two values above. 


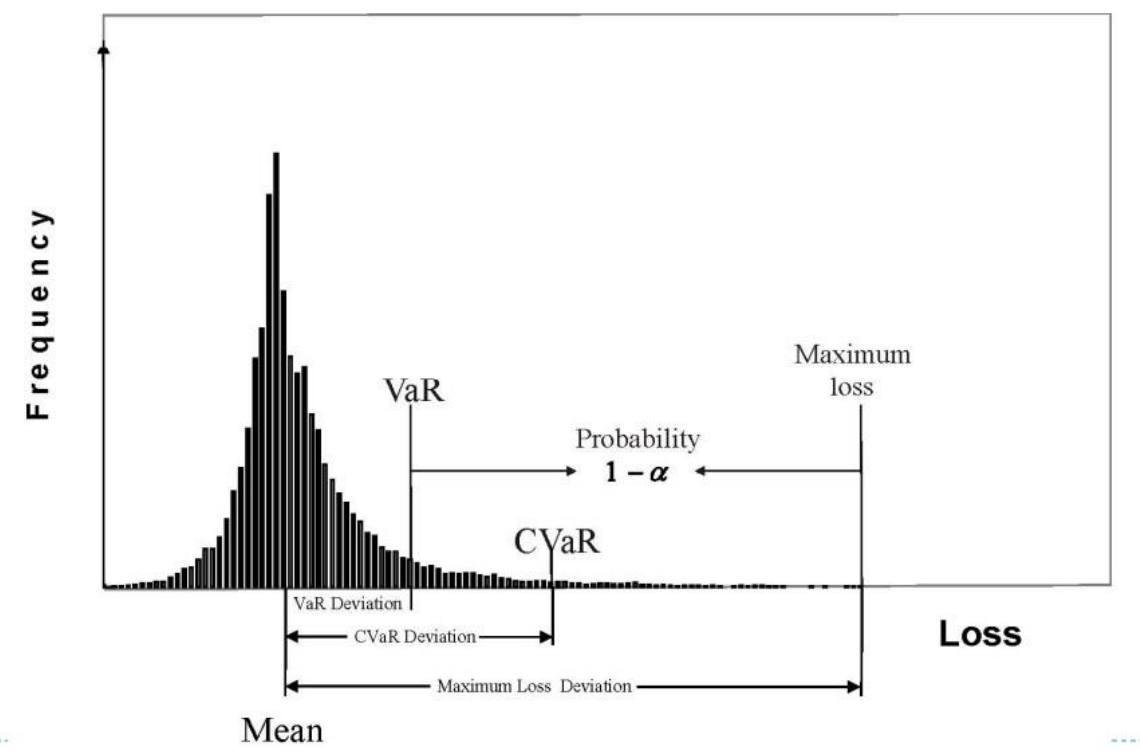

Figure 4: difference between VaR and CVaR

To conclude this Section, it is worthy stressing that also the CVar Method summarizes the information bring by a CDF in a point, as other works of the literature (e.g., [49]). Nonetheless, the CVar point has a clear engineering interpretation, which directly relates to the final decisions.

\section{Case Study}

In this paper, we consider a real practical case study concerning the maintenance optimization of a gas turbine nozzle system affected by different degradation mechanisms. The degradation of the nozzles is modelled as a four-state degradation model, in which the transitions can occur from one degradation state to the next degraded state only, and the stochastic transition times between the states obey Weibull distributions.

Recall that the scope of the case study is to investigate the potential of the proposed techniques in optimizing the condition Based Maintenance (CBM) policy applied to the nozzle system, while giving due account to the uncertainty in the parameter estimates. For this, we consider a case study derived from a real industrial application faced by the authors, but with arbitrarily chosen values of the maintenance model parameters.

The data available to estimate the parameters of the stochastic model are the outcomes of opportunistic, non-periodic, visual inspections of the turbine nozzles. That is, upon inspection, the maintenance experts disassemble the turbine and check the nozzles health states to qualitatively classify them into 'Good', 'Light', 'Medium' and 'Heavy' (Figure 5). In particular, every nozzle system is made up of $N=22$ nozzles. 


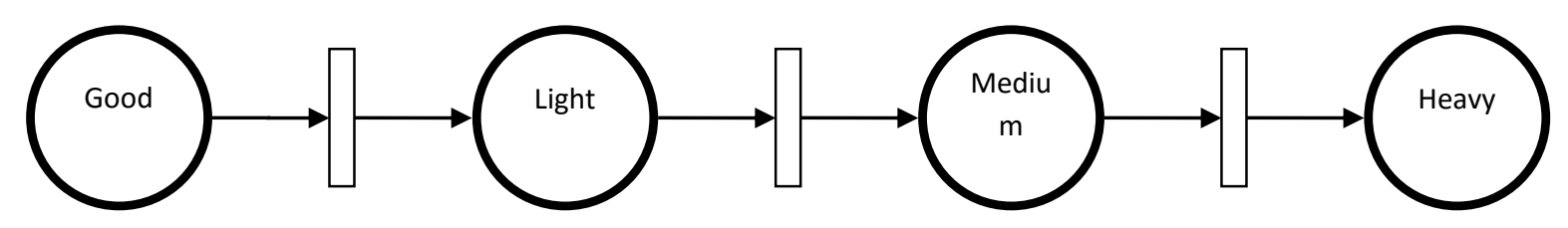

Figure 5: Scheme of the degradation mechanism

These data are used to estimate the parameters $\alpha_{k}, \beta_{k}$ of the Weibull distribution of the transition $T_{k}$ from state $S_{k}$ to state $S_{k+1}, k=1, \ldots, 3$, together with the corresponding uncertainties. For simplicity, we assume that such epistemic uncertainty in the parameter values is represented by normal distributions centered on the parameter ML estimates and with a given standard deviations. The general methodology to estimate these quantities can be found in [13].

\begin{tabular}{|c|c|c|c|c|c|c|c|c|c|c|c|}
\hline \multicolumn{2}{|c|}{$\alpha_{1}$} & \multicolumn{2}{|c|}{$\beta_{1}$} & \multicolumn{2}{|c|}{$\alpha_{2}$} & \multicolumn{2}{|c|}{$\beta_{2}$} & \multicolumn{2}{|c|}{$\alpha_{3}$} & \multicolumn{2}{|c|}{$\beta_{3}$} \\
\hline Value & $\Sigma$ & Value & $\sigma$ & Value & $\sigma$ & Value & $\sigma$ & Value & $\sigma$ & Value & $\sigma$ \\
\hline \multirow{2}{*}{1.38} & 0.014 & \multirow{2}{*}{0.71} & 0.007 & \multirow{2}{*}{12.73} & 0.016 & \multirow{2}{*}{0.16} & 0.029 & \multirow{2}{*}{6.18} & 0.014 & \multirow{2}{*}{0.65} & 0.017 \\
\hline & 0.084 & & 0.053 & & 0.097 & & 0.045 & & 0.083 & & 0.047 \\
\hline
\end{tabular}

Table 1: values of the Weibull parameters for the transitions between helth states.

In particular, to test the potential of the proposed GA advancements in treating uncertain fitnesses, we consider two numerical settings, with different amount of uncertainty affecting the estimates (Table 1). Namely, for every parameter in Table 1, the first column reports the MLE, whereas two different values of the standard deviations of the corresponding ML estimators are reported in the rows of the second column.

The CBM approach applied to the nozzle system under study is based on the continuous monitoring of the turbine efficiency by processing the information provided by sensors which trace physical variables such as pressure, temperature, etc. When the efficiency value drops below a given threshold $T_{E}$, then the nozzle system is replaced. Replacement makes the system unavailable for $U_{R}=2$ days. The cost of the consequent business interruption is given by the product of the duration of the unavailability period times the annual income $I$, which is defined as the income corresponding to one year of turbine continuous, full capacity operation. In this paper $I=20 M €$. Thus, the total $\operatorname{cost} C_{R}$ associated to a replacement action upon the achievement of $T_{E}$ is the sum of the business interruption cost due to system unavailability and the $\operatorname{cost} C_{S}=3 M €$ of replacing the nozzle system.

The nozzle system is also periodically inspected, with period $\Pi$. Every inspection is performed by one maintenance operator, who takes $T_{i n s p}=8$ days for carrying out the machine disassembling and re-assembling operations necessary to check the health state of the nozzles. Obviously, larger values of $\Pi$ steer the policy towards a full exploitation of the components and avoid ineffective machine 
stops. On the contrary, smaller values of $\Pi$ allow the machine operation in better health conditions with larger efficiency values. For this reason, $\Pi$ is an influential decision variable to optimize the maintenance policy.

The duration $t\left(S^{\gamma}\right)$ of the preventive maintenance action performed on component $\gamma$ depends on the degradation state $S^{\gamma}$ in which it is found (Table 2). More precisely, fixing nozzles in degradation state $S_{2}$ requires one operator working for 0.5 day; 1 day is needed for a maintenance operator to repair nozzles in degradation state $S_{3}$. Finally, if a nozzle is heavily degraded (i.e., in degradation state $S_{4}$ ), then a maintenance operator takes 3 days to repair it.

Notice that a simplifying assumption is made in this study: independently on the degradation state where the nozzles are found (light, medium, heavy), these are always repaired upon inspection (i.e., not replaced), and their conditions after maintenance are always considered as good as new (AGAN).

\begin{tabular}{|lc|}
\hline & $t\left(\boldsymbol{S}^{\gamma}\right)[$ days $]$ \\
\hline $\boldsymbol{S}^{\gamma}=\boldsymbol{S}_{\mathbf{2}}$ & 0.5 \\
\hline $\boldsymbol{S}^{\gamma}=\boldsymbol{S}_{\mathbf{3}}$ & 1 \\
\hline $\boldsymbol{S}^{\gamma}=\boldsymbol{S}_{\mathbf{4}}$ & 3 \\
\hline
\end{tabular}

Table 2: values of the duration of the maintenance actions

From this, it appears that the preventive maintenance time $T_{M}$ required for repairing all the $N$ nozzles is given by:

$$
T_{M}=T_{i n s p}+\sum_{\gamma=1}^{N} t\left(S^{\gamma}\right)
$$

Obviously, the system is unavailable during inspections and repairs. This causes a business interruption, whose cost is given by the part of the annual income $I$ that the maintenance actions prevent from being gained. Thus, reducing the amount of time spent in repairing the nozzle system has a beneficial effect on the maintenance costs. In this respect, a larger number of maintenance operators $\boldsymbol{N}_{\boldsymbol{m o}}$ can be involved in repairing actions.

The effect on the time reduction is given by:

$$
T_{M}=T_{i n s p}+\frac{\sum_{\gamma=1}^{N} t\left(S^{\gamma}\right)}{N_{m o}}
$$

On the other side, reducing maintenance time has its own cost, as maintenance operators must be paid for their work. We assume that their daily cost is $C_{O}=2000 \frac{€}{\operatorname{man}}$.

Then, $N_{m o}$ is another decision variable that enters the optimization of the CBM policy. 
Generally speaking, nozzle degradation entails loss in turbine efficiency, whose magnitude depends on the degradation state. In this work, we assume that when the generic nozzle $\gamma$ enters degradation state $S_{2}$, it causes a loss $l_{E}\left(S^{\gamma}=S_{2}\right)=0.2 \%$ in turbine efficiency. An additional drop of $0.3 \%$ is associated to each component in degradation state $S_{3}$ (i.e., $l_{E}\left(S^{\gamma}=S_{3}\right)=0.3 \%$ ), whereas each nozzle in state $S_{4}$ brings about a further, large loss of $0.5 \%$ (i.e., $l_{E}\left(S^{\gamma}=S_{4}\right)=0.5 \%$ ).

\begin{tabular}{|ll|}
\hline & $\boldsymbol{l}_{E}\left(\boldsymbol{S}^{\gamma}\right)$ \\
\hline$S^{\gamma}=S_{2}$ & $0.2 \%$ \\
\hline$S^{\gamma}=S_{4}$ & $0.3 \%$ \\
\hline
\end{tabular}

Table 3: values of the loss of efficiency

Thus, the loss $L_{E}$ in turbine efficiency in a cycle (i.e., the time between two maintenance actions) is given by:

$$
L_{E}=\sum_{\gamma=1}^{N} \sum_{k=1}^{3} l_{E}\left(S^{\gamma}=S_{k+1}\right)\left(T_{\text {stop }}-T_{k+1}^{\gamma}\right)
$$

where $T_{\text {stop }}$ is the end of the cycle (i.e., the inspection time at the end of the interval $\Pi$ or the time in which the turbine efficiency reaches the threshold $T_{E}$, whichever comes first), $T_{k}^{\gamma}$ is the stochastic transition time $T_{k}$ of component $\gamma$, from state $S_{k}$ to state $S_{k+1}$

Notice that the simplified scheme considered in this work entails that the worst condition (i.e., the $N=22$ nozzles are all in degradation state $S_{4}$ ) determines a total loss in turbine efficiency of at most $22 *(0.2+0.3+0.5) \%=22 \%$.

Turbine inefficiency entails a cost, which is due to the production loss with respect to the full capacity production conditions. This is given by the part of the annual income that inefficiency prevents from being gained. That is, $I_{C}=L_{E} \cdot I$ is the inefficiency cost in a cycle.

To sum up, the maintenance model described allows estimating the values of cost and unavailability corresponding to a triplet of decision variables $T_{E}, N_{m o}$ and $\Pi$.

As mentioned before, a double Monte Carlo algorithm has been implemented to propagate the uncertainty from the degradation model parameters to the maintenance performance indicators, where the internal Monte Carlo loop simulates the life process of the turbine nozzles over a fixed time horizon (i.e., the aleatory uncertainty), whereas the external loop is used to sample the parameters of the degradation model (i.e., epistemic uncertainty).

Finally, for clarity, all the parameters and variables of the case study, with relevant explanations, values and formulas, are summarized in Table 4. 


\begin{tabular}{|c|c|c|}
\hline Symbol & Description & Value \\
\hline$C_{o}$ & Maintenance operator daily cost & $2000 €$ \\
\hline$C_{S}$ & Cost for replacing the nozzle system & $3000000 €$ \\
\hline$C_{R}$ & $\begin{array}{c}\text { Total Cost for replacing the nozzle } \\
\text { system }\end{array}$ & $C_{S}+U_{R} \cdot I$ \\
\hline$I_{C}$ & Inefficiency cost in a cycle & $L_{E} \cdot I$ \\
\hline I & Annual Income & $20000000 €$ \\
\hline$\Pi$ & Inspection Interval & Decision variable \\
\hline $\boldsymbol{l}_{\boldsymbol{E}}\left(\boldsymbol{S}^{\gamma}=\boldsymbol{S}_{\boldsymbol{k}}\right)$ & $\begin{array}{l}\text { Loss in turbine efficiency when } \\
\text { component } y \text { enters degradation state } \\
S_{k}\end{array}$ & See Table 3 \\
\hline$L_{E}$ & Loss in turbine efficiency in a cycle & $L_{E}=\sum_{\gamma=1}^{N} \sum_{i=1}^{3} l_{E}\left(S^{\gamma}=S_{i}\right)\left(T_{\text {stop }}-T_{i}^{\gamma}\right)$ \\
\hline $\boldsymbol{N}$ & $\begin{array}{c}\text { Number of similar components in the } \\
\text { system }\end{array}$ & 22 \\
\hline$N_{m o}$ & $\begin{array}{l}\text { Number of maintenance operators } \\
\text { involved in repairing actions }\end{array}$ & Decision variable \\
\hline $\boldsymbol{p}$ & $\begin{array}{c}\text { Number of preventive maintenance } \\
\text { interventions }\end{array}$ & $\begin{array}{l}\text { Random variable, which depends on the } \\
\text { simulated Turbine hystory }\end{array}$ \\
\hline $\boldsymbol{R}$ & Number of replacements during $T_{H}$ & $\begin{array}{l}\text { Random variable, which depends on the } \\
\text { simulated Turbine hystory }\end{array}$ \\
\hline$S_{k}$ & $k$ - th degradation state & $k=1, \ldots, 4$ \\
\hline$S^{\gamma}$ & $\begin{array}{l}\text { Variable indicating the degradation state } \\
\text { of component } \gamma\end{array}$ & $\gamma=1, \ldots, 3$ \\
\hline $\boldsymbol{t}\left(\boldsymbol{S}^{\gamma}=\boldsymbol{S}_{\boldsymbol{k}}\right)$ & $\begin{array}{l}\text { Time to repair nozzle } y \text { when it is found } \\
\text { in state } S_{k}\end{array}$ & See Table 2 \\
\hline$T_{E}$ & Efficiency Threshold & Decision Variable \\
\hline$T_{H}$ & Time Horizon & 10 years \\
\hline$T_{\text {insp }}$ & Duration of inspections & 8 days \\
\hline$T_{k}^{\gamma}$ & $\begin{array}{l}\text { Stochastic transition time } T_{k} \text { from } S_{k} \text { to } \\
S_{k+1} \text { experienced by component } v\end{array}$ & \\
\hline$T_{\text {stop }}$ & Time instants at which the cycle ends & $\begin{array}{c}\text { Either the inspection time at the end of } \\
\text { the interval II or the time in which the } \\
\text { turbine efficiency reaches the threshold } \\
T_{E} \text {, whichever comes first }\end{array}$ \\
\hline$T_{M}$ & $\begin{array}{l}\text { Duration of preventive maintenance } \\
\text { actions }\end{array}$ & $T_{M}=T_{i n s p}+\frac{\sum_{\gamma=1}^{N} t\left(S^{\gamma}\right)}{N_{m o}}$ \\
\hline$U_{R}$ & $\begin{array}{l}\text { System Unavailability owing to the } \\
\text { replacement task }\end{array}$ & 2 days \\
\hline $\boldsymbol{U}$ & $\begin{array}{c}\text { System Unavailability (objective } \\
\text { function) }\end{array}$ & $U=E\left[\frac{p \cdot T_{E}+R \cdot U_{R}}{T_{H}}\right]$ \\
\hline
\end{tabular}

Table 4: Case study parameters and variables

\section{Results}

In this Section, the extended NSGA-II algorithm proposed in Section 3 is applied to optimize the CBM policy described in Section 4. That is, we are looking for the set of combinations of the three variables $\Pi, T_{E}$ and $N_{m o}$ that minimizes the cost and system unavailability over a time horizon of 10 years. Table 5 summarizes the characteristics of the search space in which the optimal solution is sought, where the number of bits indicates the number of points in which the search intervals are divided $\left(2^{n \_b i t s}\right)$. 


\begin{tabular}{|ccc|}
\hline Decision Variable & Search Space & Number of bits \\
\hline $\boldsymbol{\Pi}$ [inspection period, years] & {$[5,10]$} & 6 \\
\hline $\boldsymbol{T}_{\boldsymbol{E}}[\%$ efficiency] & {$[10,20]$} & 5 \\
\hline $\boldsymbol{N}_{\boldsymbol{m o}}$ [number of operators] & {$[15,23]$} & 3 \\
\hline
\end{tabular}

Table 5: Genetic algorithm search space

First, the extended NSGA-II algorithm has been run in case of small uncertainty in the degradation model parameters, in the setting summarized in Table 6. The results relative to a run of 10 generations are summarized in Appedix A, which reports the values of the decision variables corresponding to the optimal Pareto set.

\begin{tabular}{|cc|}
\hline Genetic algorithm parameters & Search setting \\
\hline Population size H & 80 \\
\hline Number of Generations & 10 \\
\hline Selection & Binary Tournament Selection \\
\hline Replacement & Random \\
\hline Mutation Probability & 0.2 \\
\hline Crossover Probability & 1 \\
\hline Number of Mc Simulation & $200 \times 500$ \\
\hline
\end{tabular}

Table 6: NSGA-II search setting

The solutions in Appendix A are all characterized by large values of the numbers of operators $N_{m o}$ performing the maintenance actions. This means that setting such decision variable to smaller values entails a significant worsening in the maintenance policy performance, due to large system unavailability.

Moreover, the solutions found by the algorithm can be grouped into two different classes:

1) The first class, rows $1-4$, sets the preventive inspection at five years (i.e., half time horizon) and uses the threshold to monitor the efficiency of the plant. This setting consistently reduces the costs, but entails larger unavailability values (bottom-right part in Figure 6).

2) The second group (remaining rows) relies on the efficiency threshold $T_{E}$ to stop the turbine, only. In fact, $\Pi$ is fixed to almost 10 years, which entails that the turbine is never inspected. Contrarily to the first solutions, this consistently reduces the unavailability, but leads to larger costs.

In Figure 6, the Optimal Pareto Front is reported: horizontal and vertical lines represent the range between the fifth and ninety-fifth percentiles of the CDFs of unavailability and cost, respectively, whereas the line crosses identify the means of the two distributions. 


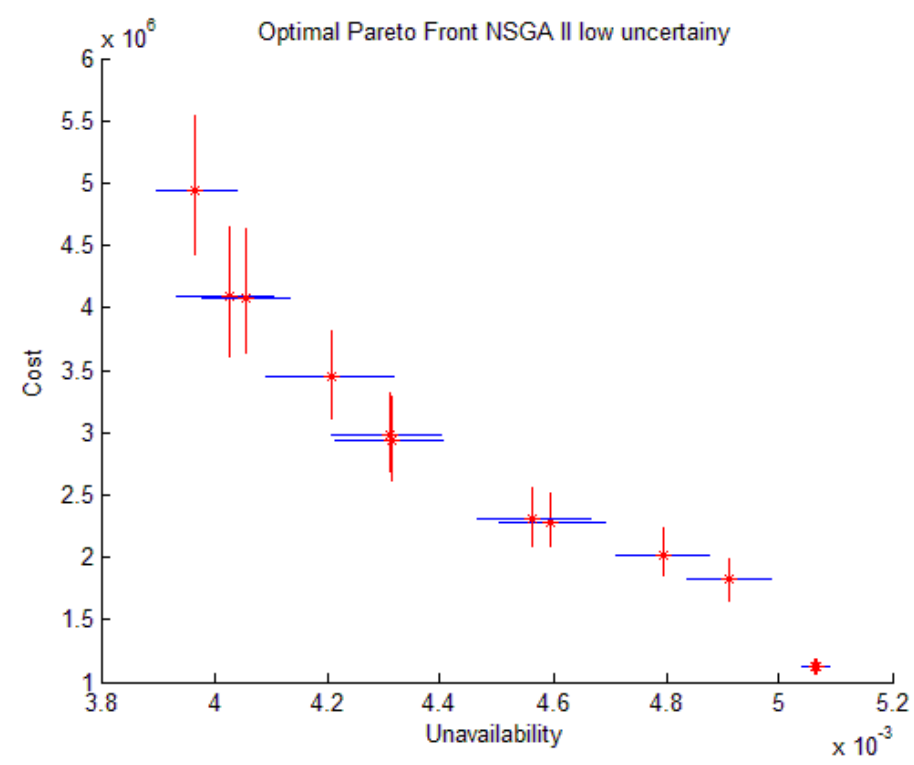

Figure 6: NSGA-II Optimal Pareto Front: horizontal and vertical lines are the 5-th and 95th percentiles of the unavailability and cost distributions.

In the second uncertainty setting, i.e., with larger standard deviations (Table 1), the algorithm provides the results reported in Appendix B.

Comparing these solutions with those in Appendix A, it emerges that larger uncertainty values increase the cardinality of the Pareto Optimal Set. This is an expected result; roughly speaking, larger uncertainties lead to more 'lengthened' CDFs (e.g., larger ranges between the fifth and ninety-fifth percentiles of cost and unavailability), and thus increase the number of non-dominated solutions. This result also highlights the added value of considering optimization algorithms that give full account to uncertainty. That is, these algorithms give the possibility of considering among the Pareto optimal set, solutions that would have been otherwise discarded.

Figure 7 reports the Pareto front corresponding to the Pareto set in Appendix B, with the 90\% confidence intervals. Notice that, the bands describing the uncertainty in the costs are larger in correspondence of smaller values of unavailability. This is due to the larger variability of the repair costs when the turbine is periodically inspected, owing to the variability of the degradation state in which the nozzles can be found. On the contrary, when the turbine is stopped based on $T_{E}$, the health conditions of the nozzles are similar to each other in the different stochastic simulations. 


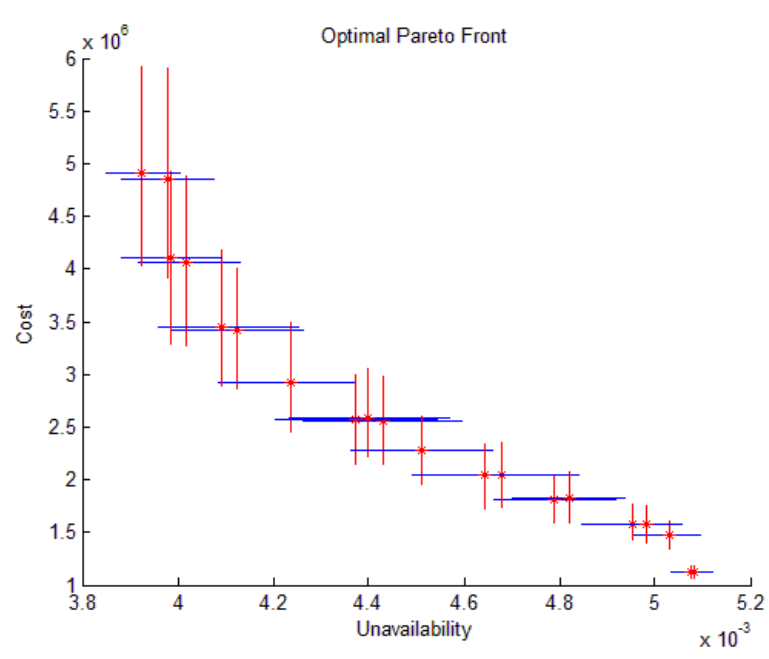

Figure 7: NSGA-II Optimal Pareto Front, in case of larger epistemic uncertainty.

Finally, a comment seems in order about the choice of stopping the optimization algorithm at $G=10$ generations. In this case study, such choice is justified by the fact that the solutions found by the extended NSGA-II algorithm belong to the two classes described above, which are the same provided by the CVaR method. This makes us confident that even if the results do not belong to the actual optimal Pareto set, they are not far from it.

More generally, setting the value of $G$ or adopting a different stopping criterion remains a critical issue, especially in the light of the findings of [57], where the authors showed that to reduce the computational times, investing on a large number of generations (i.e., "evolution" [57]) is to be preferred to performing a large number of Monte Carlo runs (i.e., "accuracy"). This issue has not been answered by this work, and will be tackled in future research works. A possibility is to adapt the hyper-volume stopping criterion [32] to the case of uncertain fitness values (i.e., stop the evolution when the shape of the Pareto front remains constant) or perform a cluster analysis on the Pareto front [60], or analyse the influence of increasing $G$ on the search to derive a novel stopping criterion.

\subsection{Hybrid NSGA-II and CVaR measure}

In this Section, the $C V a R_{\alpha}$ risk measure is introduced in the objective functions of the NSGA-II algorithm. Specifically, we use Equations (1) and (2) as objective functions with three different values of the parameter $\beta: 0,0.5,1$, and percentile $\alpha=0.7$. Also in this case, we first consider the numerical setting in which the parameters are affected by a small amount of uncertainty. The setting of the remaining parameters of the NSGA-II are reported in Table 6.

Appendix $\mathrm{C}$ lists the solutions of the Pareto optimal set and the corresponding objective function values, whereas Figure 8 shows the Pareto optimal fronts for the different values of $\beta$. 

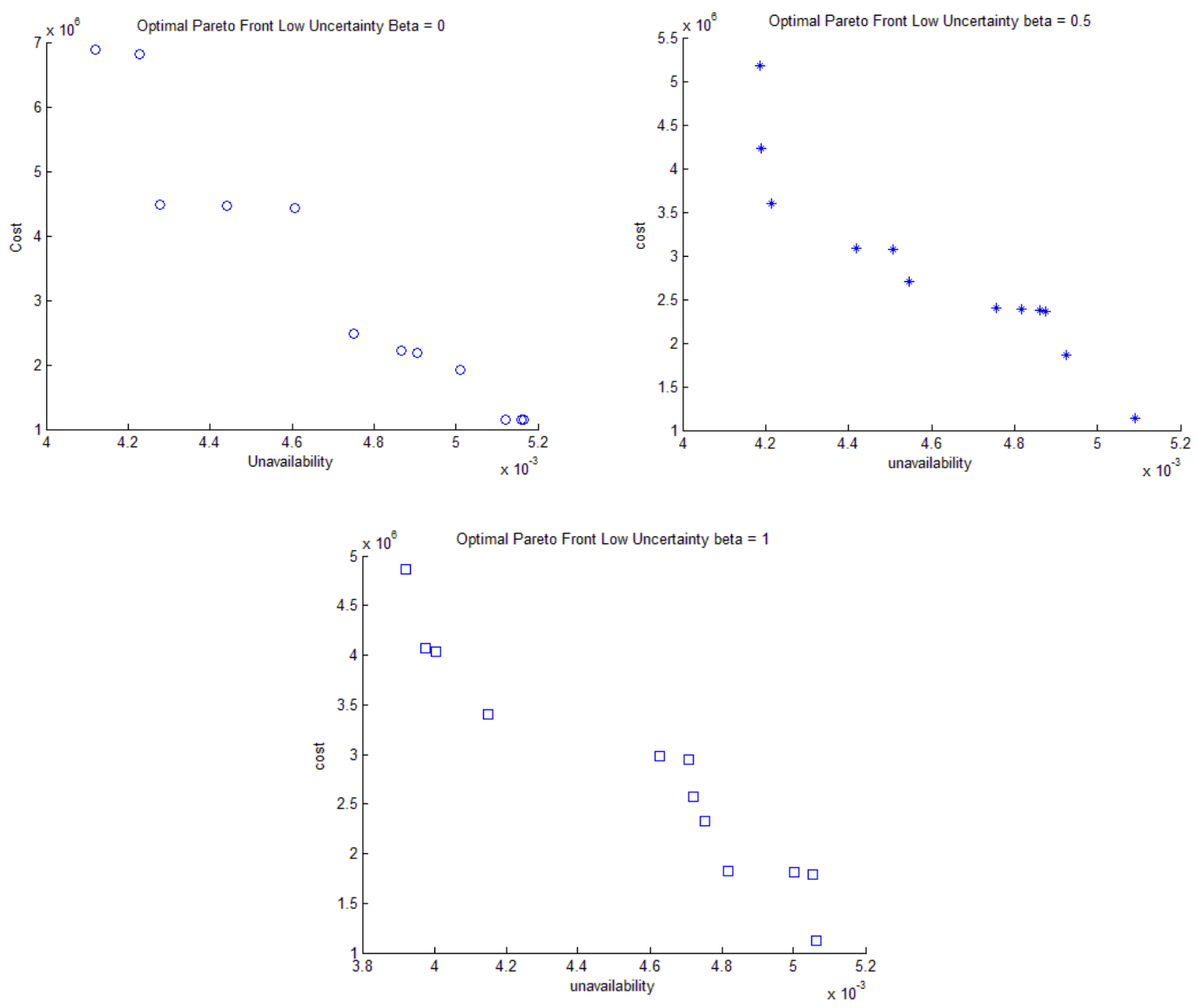

Figure 8: Pareto Optimal Front relative to $\beta=0,0.5$ and 1, in case of smaller epistemic uncertainty.

From Figure 8, it emerges that the larger the value of $\beta$ the smaller the costs. This is due to the fact that smaller values of $\beta$ drive the multi objective optimization to focusing on the expected values of the objectives, whereas larger values of $\beta$ put emphasis on the level of risk $\left(C V a R_{\alpha}\right)$ associated to the solutions. This result cannot be appreciated on the unavailability objective function, as its variability is very small.

On the other side, whichever the value of $\beta$ is, the solutions provided by the CVaR measure are always belonging to the same two groups identified by the NSGA-II algorithm. This result also holds when we have an increase in the values of the variance of the estimators of the degradation model parameters, as it can be easily seen from Appendix D and Figure 9, which show the Pareto optimal set and the corresponding Pareto optimal front. However, although the investigated algorithms give similar solutions, there are significant differences between them: the NSGA-II proposed in this work gives full account to the uncertainty in the objective functions, as it handles directly the CDFs describing such uncertainty. On the contrary, the CVaR technique summarizes the CDFs in single points, i.e., the average values of the risky parts of these distributions, with consequent loss of information. On the other side, this simplification allows for a significant improvement in the 
interpretability of the results of the algorithm: the maintenance decision maker knows that the optimal solutions provided by the algorithm correspond to a given risk level of having poorer unavailability and cost performances, which is also set by himself/herself. In this respect, different algorithms can be applied to help the decision maker in making the final choice when the hybrid NSGA-II and CVaR approach is adopted [59], which cannot be easily applied when using the extended NSGA-II, as they rely on the geometrical properties of the Pareto front. The development of methods to apply the concept of geometrical distance to the Pareto fronts depicted in Figure 7 will be the focus of future research work.

Moreover, the fact that the solutions obtained for $\beta=0$ (i.e. pure $C V a R_{\alpha}$ ) and $\beta=1$ (i.e., average fitness values) are similar to each other may lead to conclude that there is no added value in considering the uncertainty in the fitnesses. However, this conclusion is generally wrong; in fact, there are optimization problem in which the Pareto optimal sets are different in correspondence of different values of $\beta$ values [41]. In this respect, notice also that the comparison of Figure 8 and Figure 9 highlights a change in the shape of the Pareto front: the front appears to be more swollen in case of larger values of the variance of the ML estimators. This is due to the fact that larger uncertainty in the model parameters moves the CVaR values of the solutions toward larger values.

Yet, to compare the extended NSGA-II method with the hybrid NSGA-II and CVaR approaches, we also consider their computational times (Table 6), relevant to 10 generations of 80 individuals. From Table 7, it emerges that using the CVaR to summarize the uncertainty in the fitnesses allows for smaller computational times, as we have to look at a single value rather than to the entire distribution. Notice also that the computational times are very large because we need applying the computational burdensome double-loop Monte Carlo procedure for every individual at any generation, and also the time-consuming ranking procedure proposed in Section 2.

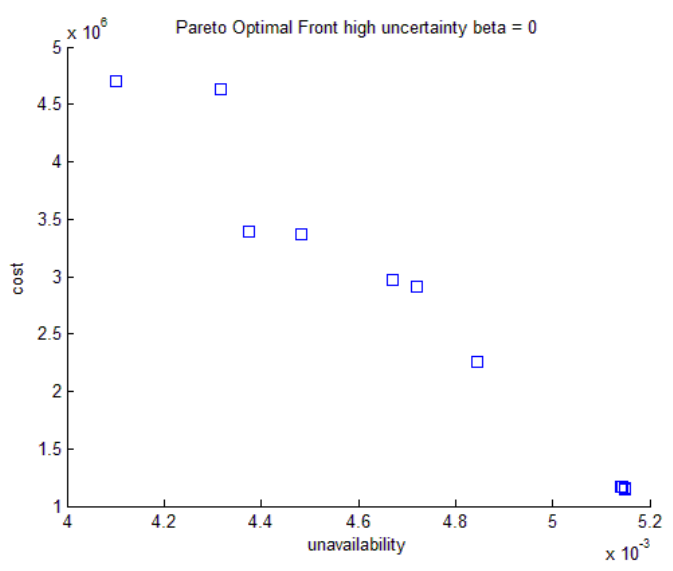



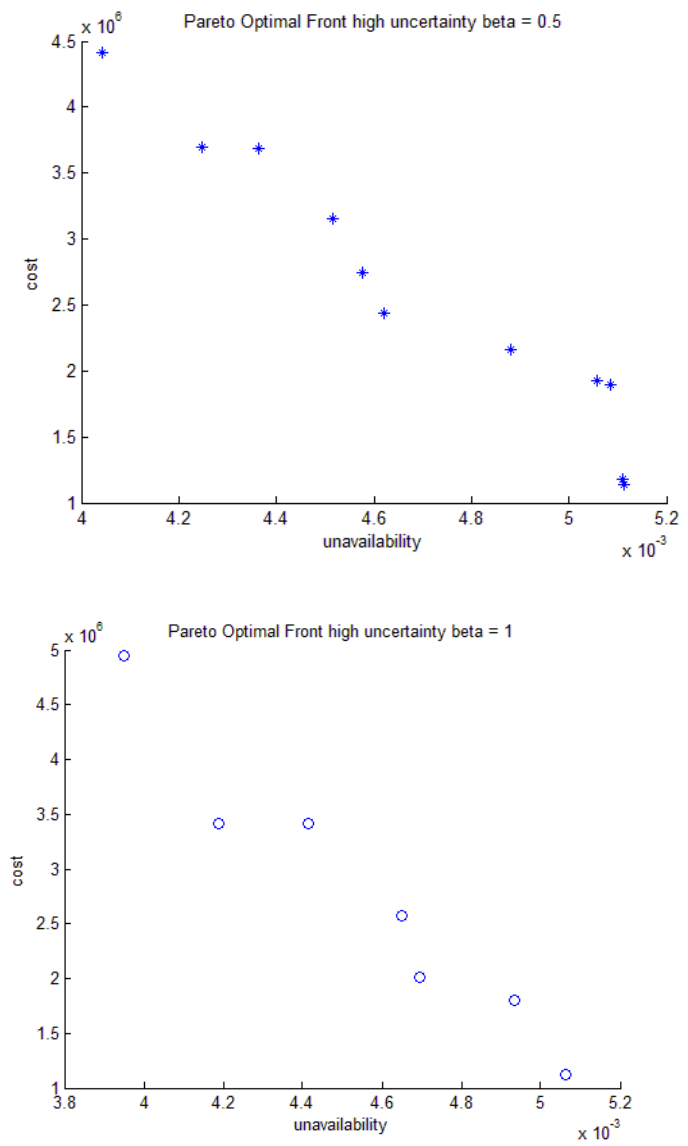

Figure 9: Pareto front relative to $\beta=0,0.5$ and 1 , in case of larger epistemic uncertainty.

\begin{tabular}{|c|c|}
\hline Algorithm & Computational Time \\
\hline NSGA-II & 20 hours \\
\hline CVaR & 6 hours \\
\hline
\end{tabular}

Table 7: Computational Time for the methods applied, on an Intel Core $17 \mathrm{CPU}, 1.8 \mathrm{GHz}$, machine

\section{Conclusion}

In this work, two techniques based on the Multi Objective Genetic Algorithms have been proposed to solve the problem of CBM affected by uncertainty, which both rely on an innovative algorithm for sorting probability distributions and on a new definition of Pareto dominance. The first technique is based on the NSGA-II algorithm, whereas the second is a combination of NSGA-II and CVaR index. These methods have been applied to a practical case study, providing similar results. Nonetheless, there are significant differences between these approaches: the NSGA-II gives full account to the uncertainty in the objective functions, as it handles the CDFs describing such uncertainty; on the contrary, the $\mathrm{CVaR}$ technique summarizes the CDFs in single points, i.e., the average values of the risky parts of these distributions. On one side, the simplification introduced by the CVaR algorithm leads to a loss of information. On the other side, this allows for a significant simplification of the 
algorithm and provides the maintenance decision maker with more easily understandable results, as he/she knows that the optimal solutions provided by the algorithm correspond to a given risk level, also set by the decision maker.

Additional findings of the work are:

- The Pareto optimal set depends on the uncertainty in the model parameters. That is, the larger the uncertainty the more swollen the Pareto front.

- The computational times of the hybrid NSGA-II and CVaR method are considerably better than those of the extended NSGA-II.

Future research works will focus on investigating the effects of the number of generations $G$ on the results of the search, and devising different stopping criteria.

\section{Acknowledgment}

The authors would like to thank the anonymous reviewers for their valuable comments and suggestions to improve the paper.

\section{Appendix}

\section{A. Pareto Optimal set of NSGA II}

\begin{tabular}{|ccc|}
\hline$\Pi$ & $T_{E}$ & $N_{m o}$ \\
\hline 5.0000 & 15.6250 & 22 \\
\hline 5.0000 & 17.8125 & 22 \\
\hline 5.0000 & 19.0625 & 22 \\
\hline 5.0781 & 19.0625 & 22 \\
\hline 9.7656 & 14.3750 & 21 \\
\hline 9.7656 & 14.6875 & 21 \\
\hline 9,8438 & 13.4375 & 21 \\
\hline 9.8438 & 14.0625 & 21 \\
\hline 9,9219 & 12.5000 & 20 \\
\hline 9.9219 & 12.8125 & 19 \\
\hline 9.9219 & 12.8125 & 20 \\
\hline 9.9219 & 13.1250 & 18 \\
\hline 9.9219 & 13.1250 & 19 \\
\hline 9.9219 & 13.4375 & 21 \\
\hline 9.9219 & 14.3750 & 16 \\
\hline 9.9219 & 14.0625 & 20 \\
\hline 9.9219 & 19.1250 & 19 \\
\hline
\end{tabular}

\section{B. Pareto Optimal set of NSGA-II with high uncertainty}




\begin{tabular}{|c|c|c|}
\hline II & $T_{E}$ & $N_{m o}$ \\
\hline 5.0000 & 15.6250 & 22 \\
\hline 5.0000 & 17.8125 & 22 \\
\hline 5.0000 & 19.0625 & 22 \\
\hline 5.2344 & 17.5000 & 22 \\
\hline 5.3125 & 17.5000 & 22 \\
\hline 5.3125 & 18.7500 & 22 \\
\hline 9.8438 & 13.7500 & 22 \\
\hline 9.8438 & 14.6875 & 22 \\
\hline 9.8438 & 15.0000 & 22 \\
\hline 9.9219 & 12.5000 & 22 \\
\hline 9.9219 & 12.8125 & 21 \\
\hline 9.9219 & 12.8125 & 22 \\
\hline 9.9219 & 13.1250 & 21 \\
\hline 9.9219 & 13.1250 & 22 \\
\hline 9.9219 & 13.4375 & 22 \\
\hline 9.9219 & 13.7500 & 21 \\
\hline 9.9219 & 13.7500 & 22 \\
\hline 9.9219 & 14.0625 & 22 \\
\hline 9.9219 & 14.3750 & 21 \\
\hline 9.9219 & 14.3750 & 22 \\
\hline 9.9219 & 14.6875 & 22 \\
\hline 9.9219 & 15.0000 & 22 \\
\hline 9.9219 & 15.3125 & 18 \\
\hline 9.9219 & 15.3125 & 22 \\
\hline
\end{tabular}

\section{NSGA-II \& CVaR, in case of smaller epistemic uncertainty.}

\begin{tabular}{|c|c|c|c|c|c|}
\hline Value of $\beta$ & $\Pi$ & $T_{E}$ & $N_{m o}$ & $f_{1}$ & $\boldsymbol{f}_{2}$ \\
\hline \multirow{12}{*}{$\beta=0$} & 5.0000 & 19.0625 & 22 & 0.005119 & $1.15725 \mathrm{e} 6$ \\
\hline & 5.0781 & 17.5000 & 21 & 0.005158 & $1.15566 \mathrm{e} 6$ \\
\hline & 5.1562 & 15.9375 & 21 & 0.005165 & $1.15336 \mathrm{e} 6$ \\
\hline & 9.4531 & 12.8125 & 19 & 0.004606 & $4.42799 \mathrm{e} 6$ \\
\hline & 9.6093 & 12.1875 & 22 & 0.004228 & $6.82013 e 6$ \\
\hline & 9.6875 & 14.3750 & 22 & 0.004906 & $2.18279 \mathrm{e} 6$ \\
\hline & 9.6875 & 14.6875 & 22 & 0.005011 & $1.92226 \mathrm{e} 6$ \\
\hline & 9.7656 & 12.8125 & 15 & 0.004442 & $4.46420 \mathrm{e} 6$ \\
\hline & 9.7656 & 12.8125 & 19 & 0.004278 & $4.48690 \mathrm{e} 6$ \\
\hline & 9.8437 & 12.1875 & 20 & 0.004121 & $6.89112 \mathrm{e} 6$ \\
\hline & 9.8437 & 14.0625 & 20 & 0.004750 & $2.47483 \mathrm{e} 6$ \\
\hline & 9.9218 & 14.3750 & 19 & 0.004865 & $2.22316 \mathrm{e} 6$ \\
\hline \multirow{5}{*}{$\beta=0.5$} & 5.0000 & 15.9375 & 22 & 0.005091 & $1.13765 \mathrm{e} 6$ \\
\hline & 9.5312 & 14.0625 & 22 & 0.004875 & $2.36447 \mathrm{e} 6$ \\
\hline & 9.6875 & 14.0625 & 19 & 0.004861 & $2.37491 \mathrm{e} 6$ \\
\hline & 9.6875 & 14.0625 & 20 & 0.004816 & $2.39103 e 6$ \\
\hline & 9.7656 & 14.6875 & 22 & 0.004925 & $1.85614 \mathrm{e} 6$ \\
\hline
\end{tabular}




\begin{tabular}{|c|c|c|c|c|c|}
\hline & 9.8437 & 12.5000 & 16 & 0.004185 & $5.17130 \mathrm{e} 6$ \\
\hline & 9.8437 & 12.8125 & 18 & 0.004189 & $4.23272 \mathrm{e} 6$ \\
\hline & 9.8437 & 13.1250 & 22 & 0.004215 & $3.59780 \mathrm{e} 6$ \\
\hline & 9.8437 & 13.4375 & 18 & 0.004506 & $3.06899 \mathrm{e} 6$ \\
\hline & 9.8437 & 13.7500 & 20 & 0.004547 & $2.70590 \mathrm{e} 6$ \\
\hline & 9.9218 & 13.4375 & 18 & 0.004419 & $3.09173 \mathrm{e} 6$ \\
\hline & 9.9218 & 14.0625 & 17 & 0.004757 & $2.40321 \mathrm{e} 6$ \\
\hline \multirow{13}{*}{$\beta=1$} & 5.0000 & 16.5625 & 22 & 0.005063 & $1.12333 \mathrm{e} 6$ \\
\hline & 5.0000 & 19.3750 & 22 & 0.005063 & $1.12324 \mathrm{e} 6$ \\
\hline & 9.4531 & 13.4375 & 21 & 0.004708 & $2.94671 \mathrm{e} 6$ \\
\hline & 9.4531 & 14.6875 & 22 & 0.005052 & $1.78782 \mathrm{e} 6$ \\
\hline & 9.6093 & 13.7500 & 20 & 0.004720 & $2.57789 \mathrm{e} 6$ \\
\hline & 9.6875 & 13.4375 & 17 & 0.004626 & $2.97766 \mathrm{e} 6$ \\
\hline & 9.6875 & 14.6875 & 20 & 0.005001 & $1.81776 \mathrm{e} 6$ \\
\hline & 9.8437 & 13.1250 & 22 & 0.004151 & $3.40217 \mathrm{e} 6$ \\
\hline & 9.8437 & 14.0625 & 17 & 0.004754 & $2.32436 \mathrm{e} 6$ \\
\hline & 9.9218 & 12.5000 & 22 & 0.003921 & $4.86657 \mathrm{e} 6$ \\
\hline & 9.9218 & 12.8125 & 21 & 0.004005 & $4.03651 \mathrm{e} 6$ \\
\hline & 9.9218 & 12.8125 & 22 & 0.003976 & $4.07308 \mathrm{e} 6$ \\
\hline & 9.9218 & 14.6875 & 21 & 0.004816 & $1.82957 \mathrm{e} 6$ \\
\hline
\end{tabular}

D. NSGA-II \& CVaR results, in case of larger epistemic uncertainty

\begin{tabular}{|c|c|c|c|c|c|}
\hline Value of $\boldsymbol{\beta}$ & $\Pi$ & $T_{E}$ & $N_{m o}$ & $f_{1}$ & $f_{2}$ \\
\hline \multirow{12}{*}{$\beta=0$} & 5.1563 & 16.2500 & 22 & 0.00514 & $1.16926 \mathrm{e} 6$ \\
\hline & 5.0781 & 17.1875 & 22 & 0.00514 & $1.17448 \mathrm{e} 6$ \\
\hline & 5.2344 & 16.2500 & 22 & 0.00471 & $2.91378 \mathrm{e} 6$ \\
\hline & 5.2344 & 18.1250 & 22 & 0.00515 & $1.15717 \mathrm{e} 6$ \\
\hline & 9.9219 & 13.4375 & 19 & 0.00448 & $3.36976 \mathrm{e} 6$ \\
\hline & 9.9219 & 13.7500 & 17 & 0.00471 & $2.91378 \mathrm{e} 6$ \\
\hline & 9.9219 & 12.8125 & 16 & 0.00431 & $4.63619 \mathrm{e} 6$ \\
\hline & 9.9219 & 13.4375 & 22 & 0.00437 & $3.39289 \mathrm{e} 6$ \\
\hline & 9.9219 & 12.8125 & 22 & 0.00410 & $4.70493 e 6$ \\
\hline & 9.9219 & 13.7500 & 17 & 0.00472 & $2.91378 \mathrm{e} 6$ \\
\hline & 9.9219 & 13.7500 & 18 & 0.00467 & $2.96845 \mathrm{e} 6$ \\
\hline & 9.8438 & 14.3750 & 22 & 0.00484 & $2.26309 \mathrm{e} 6$ \\
\hline \multirow{11}{*}{$\beta=0.5$} & 5.1563 & 14.6875 & 22 & 0.00511 & $1.17841 \mathrm{e} 6$ \\
\hline & 5.2344 & 19.0625 & 22 & 0.00511 & $1.14263 \mathrm{e} 6$ \\
\hline & 9.5313 & 14.6875 & 22 & 0.05085 & $1.89593 \mathrm{e} 6$ \\
\hline & 9.6875 & 14.6875 & 20 & 0.00505 & $1.92952 \mathrm{e} 6$ \\
\hline & 9.7656 & 13.1250 & 20 & 0.00436 & $3.69015 \mathrm{e} 6$ \\
\hline & 9.7656 & 13.4375 & 20 & 0.00451 & $3.15427 \mathrm{e} 6$ \\
\hline & 9.7656 & 13.7500 & 22 & 0.00457 & $2.74118 \mathrm{e} 6$ \\
\hline & 9.8438 & 13.1250 & 22 & 0.00424 & $3.69549 \mathrm{e} 6$ \\
\hline & 9.8438 & 14.3750 & 19 & 0.00488 & $2.16130 \mathrm{e} 6$ \\
\hline & 9.9219 & 12.8125 & 22 & 0.00404 & $4.41210 \mathrm{e} 6$ \\
\hline & 9.9219 & 14.0625 & 21 & 0.00462 & $2.43571 \mathrm{e} 6$ \\
\hline \multirow{3}{*}{$\beta=1$} & 5.0781 & 19.3750 & 22 & 0.00506 & $1.12285 \mathrm{e} 6$ \\
\hline & 9.6094 & 13.7500 & 22 & 0.00465 & $2.57382 \mathrm{e} 6$ \\
\hline & 9.6875 & 13.1250 & 19 & 0.00441 & $3.41215 \mathrm{e} 6$ \\
\hline
\end{tabular}




\begin{tabular}{|lllll|}
\hline 9.6875 & 14.6875 & 22 & 0.00493 & $1.79697 \mathrm{e} 6$ \\
\hline 9.9219 & 12.5000 & 21 & 0.00394 & $4.95016 \mathrm{e} 6$ \\
9.9219 & 13.1250 & 19 & 0.00418 & $3.42029 \mathrm{e} 6$ \\
\hline 9.9219 & 14.3750 & 21 & 0.00469 & $2.00797 \mathrm{e} 6$ \\
\hline
\end{tabular}

\section{Reference}

[1].Alsyouf, I., "The role of maintenance in improving companies' productivity and profitability", International Journal of Production Economics, Vol. 105, pp. 70-78, 2007.

[2].Baraldi, P., Balestrero, A., Compare, M., Benetrix, L., Despujols, A., Zio, E., "A modeling framework for maintenance optimization of electrical components based on fuzzy logic and effective age", Quality and Reliability Engineering International. Vol. 29, No. 3, pp. 385-405, 2013.

[3].Baraldi, P., Compare M., Zio E. "Uncertainty treatment in expert information systems for maintenance policy assessment", Applied Soft Computing, Vol. 22, pp. 297-310, 2014.

[4].Baraldi, P., Compare, M., Despujols, A., Zio E., "Modelling the effects of maintenance on the degradation of a water-feeding turbo-pump of a nuclear power plant", Proceedings of the Institution of Mechanical Engineers, Part O: Journal of Risk and Reliability, Vol. 225 (2), pp. 169-183, 2011.

[5].Baraldi, P., Compare, M., Zio, E. "Dempster-Shafer theory of evidence to handle maintenance models tainted with imprecision", In proceedings of the 11th International Probabilistic Safety Assessment and Management Conference and the Annual European Safety and Reliability Conference Helsinki, Finland, pp. 61-70, 2012.

[6].Baraldi, P., Compare, M., Zio, E. "Uncertainty analysis in degradation modeling for maintenance policy assessment," Proceedings of the Institution of Mechanical Engineers, Part O: Journal of Risk and Reliability, Vol. 227, No. 3, pp. 267-278, 2013.

[7].Baraldi, P., Compare, M., Zio, E., "A pratical analysis of the degradation of a nuclear component with field data," in Safety, Reliability and Risk Analysis: Beyond the Horizon Proceedings of the European Safety and Reliability Conference, ESREL, pp. 1009-1014, 2013.

[8].Baraldi, P., Compare, M., Zio, E., "Component Ranking by Birnbaum Importance in Presence of Epistemic Uncertainty in Failure Event Probabilities," IEEE Transactions On Reliability Vol. 62, No. 1, pp. 37-48, 2013.

[9].Baraldi, P., Compare, M., Zio, E., "Maintenance policy performance assessment in presence of imprecision based on Dempster-Shafer Theory of Evidence", Information Sciences, Vol. 245, pp. 112-131.

[10]. Baraldi, P., Zio, E., Compare, M., "A method for ranking components importance in presence of epistemic uncertainties,” J. Loss Prevention Process Ind., Vol. 22, no. 5, pp. 582592, 2009.

[11]. Ben-Daya, M., Duffaa, S.O., "Maintenance and quality: the missing link", Journal of Quality in Maintenance, Vol. 1, no. 1, pp. 20-26, 1995.

[12]. Coit, D.W., Jin, T., Wattanapongsakorn, N., "System optimization with component reliability estimation uncertainty: A multi-criteria approach," IEEE Transactions on Reliability, 53 (3), pp. 369-380, 2004.

[13]. Compare, M., Martini, F., Zio, E., " Semi-Markov model for erosion-corrosion degradation mechanism in gas turbine nozzle", submitted for publication.

[14]. Compare, M., Zio, E., "Genetic algorithms in the framework of Dempster-Shafer Theory of Evidence for maintenance optimization problems", accepted for publication on IEEE Reliability Transactions. 
[15]. De Cooman, G., Hermans, F., Quaeghebeur, E., "Imprecise Markov Chains And Their Limit Behaviour," Probability in the Engineering and Informational Sciences, Vol. 23, pp. 597-635, 2009.

[16]. Deb, K., Pratap, A., Agarwal, S., Meyarivan T., "A Fast and Elitist Multiobjective Genetic Algorithm: NSGA-II", IEEE transanction on evolutionary computation, Vol. 6, no. 2, 2002.

[17]. Eskandari, H., Geiger, C.D., Bird R. "Handling uncertainty in evolutionary multiobjective optimization: SPGA", Proceedings of the IEEE Congress on Evolutionary Computation, pp. 4130,4137, 2007.

[18]. $\quad$ Fan, Z.-P, Liu, Y, Feng, B. "A method for stochastic multiple criteria decision making based on pairwise comparisons of alternatives with random evaluations," European Journal of Operational Research, Vol. 207, pp. 906-915, 2010.

[19]. Furtado, M.J.H., Soares, R.F., Lins, I.D., Das Chagas Moura, M., Droguett, E.L., Firmino, P. "Multi-objective optimization of risk and cost for risk-based inspection plans" Proceedings of 11th International Probabilistic Safety Assessment and Management Conference and the Annual European Safety and Reliability Conference 2012, PSAM11 ESREL 2012, Vol. 5, pp. 4156-4167, 2012.

[20]. Ge, H., Asgarpoor, S., "Reliability Evaluation of Equipment and Substations with Fuzzy Markov Processes”, IEEE Transaction on Power System, Vol. 25, No. 3, pp. 13191328, 2010.

[21]. Giorgio, M., Guida, M., Pulcini, G., "An age- and state-dependent Markov model for degradation processes”, IIE Transactions (Institute of Industrial Engineers), Vol. 43 (9), pp. 621-632. 2011.

[22]. Greco, S., Matarazzo, B., Slowinski, R. "Rough Sets theory for multicriteria decision analysis," European Journal of Operational Research, Vol. 129, pp. 1-47, 2001.

[23]. Hughes, J.E., "Evolutionary Multi-objective Ranking with Uncertainty and Noise. In proceedings of Evolutionary Multi-Criterion Optimization”, First International Conference, EMO 2001, Zurich, Switzerland, March 7-9, pp. 329-343, 2001.

[24]. IEEE / PES Task Force, "The Present Status of Maintenance Strategies and the Impact of Maintenance on Reliability", IEEE Transactions on Power Systems. Vol. 16, no. 4, pp. 638-646, 2001

[25]. Johnson R.A, Wichern D.W., “Applied Multivariate statistical analysis”,Pearson International Edition, Sixth edition, 2007.

[26]. Knuth, D. E. "The Art of Computer Programming, Volume 3: Sorting and Searching", Addison-Wesley, 1998.

[27]. Konak, A., Coit, D.W., Smith, A.E., "Multi-objective optimization using genetic algorithms: A tutorial. Reliability Engineering and System Safety", Vol. 91, pp. 992-1007, 2006.

[28]. Kolowrocki, K., Soszynska-Budny, J., "Reliability and Safety of Complex Technical Systems and Processes: Modeling - Identification - Prediction - Optimization," Springer Series in Reliability Engineering, 2013.

[29]. Kozine, I.O., Utkin, L.V., "Interval-valued finite Markov chains", Reliable Computing, Vol. 8, No. 2, pp. 97-113, 2002.

[30]. Lehmann, E. L. "Ordered families of distributions," The Annals of Mathematical Statistics, Vol. 26, pp. 399-419, 1955.

[31]. Li, J., Kwan, R.S.K., "A fuzzy genetic algorithm for driver scheduling." European Journal of Operational Research, Vol.147, No. 2, pp. 334-344, 2003.

[32]. Li ,Y.F., Pedroni, N., Zio, E., “A Memetic Evolutionary Multi-Objective Optimization Method for Environmental Power Unit Commitment", IEE Transactions on Power Systems, 28 (3), pp. 2660-2669, 2013. 
[33]. Limbourg, P. "Multi-objective Optimization of Problems with Epistemic Uncertainty", Proceedings of Evolutionary Multi-Criterion Optimization, Third International Conference, EMO 2005, Guanajuato, Mexico, March 9-11, pp. 413-427, 2005

[34]. Lins, I.D., Droguett, E.L. "Redundancy allocation problems considering systems with imperfect repairs using multi-objective genetic algorithms and discrete event simulation," Simulation Modelling Practice and Theory, Vol. 19, No. 1, pp. 362-381, 2011.

[35]. Lisnianski, A., Levitin, G., "Multi-state system reliability: assessment, optimization and applications," World Scientific, 2003.

[36]. Lisnianski, A., Frenkel, I., Ding, Y., "Multi-state System Reliability Analysis and Optimization for Engineers and Industrial Managers," Springer, 2014.

[37]. Luce, R.D., "Semi-orders and a Theory of Utility Discrimination," Econometrica, Vol. 24, No. 2, pp. 178-191, 1956.

[38]. Marseguerra, M., Zio, E., Martorell, S., "Basics of Genetic Algorithms Optimization for RAMS Applications”, Reliability Engineering and System Safety, Vol. 91, pp. 977-991, 2006.

[39]. Martorell, S., Sanchez, A., Carlos, S., "A tolerance interval based approach to address uncertainty for RAMS+C optimization," Reliability Engineering \& System Safety, Vol. 92, No. 4, pp. 408-422, 2007.

[40]. Melnikov, A., Smirnov, I., "Dynamic hedging of conditional value-at-risk. Insurance: Mathematics and Economics", Vol. 51, No. 1, pp. 82-90, 2012.

[41]. Mena R., Hennebel M., Fu Y., Ruiz L. C., Zio E., "A Risk-Based Simulation and Multi-Objective Optimization Framework for the Integration of Distributed Renewable Generation and Storage," Renewable and Sustainable Energy Reviews, Vol. 37, pp. 778-793, 2014.

[42]. Menger, K. "Probabilistic Theory of Relations," proceedings of the National Academy of Sciences, Vol. 37, pp. 178-180, 1951.

[43]. Mitchell, M. “An Introduction to Genetic Algorithms. Cambridge," MIT Press, 1996.

[44]. Modarres, M. "Risk Analysis in Engineering: Probabilistic Techniques, Tools and

Trends," CRC Press, 2006.

[45]. Moghaddass, R., Zuo, M.J., "A parameter estimation method for a multi-state deteriorating system with incomplete information", proceeding of the Seventh International Conference on Mathematical Methods in Reliability: Theory, Methods, and Applications (MMR 2011), pp. 34-42, 2011.

[46]. Moghaddass, R., Zuo, M.J., "Multi-state degradation analysis for a condition monitored device with unobservable states", Proceedings of 2012 International Conference on Quality, Reliability, Risk, Maintenance, and Safety Engineering, ICQR2MSE 2012, pp. 549-554, 2012.

[47]. Moura, M. C.; Lins, I. D.; Droguett, E.L.; Soares, R.; Pascual, R. "A Multi-Objective Genetic Algorithm for Determining Efficient Risk-Based Inspection Programs.” Reliability Engineering \& Systems Safety, 2014

[48]. Papoulis, A. And Pillai S.U. 2002. Probability, Random Variables and Stochastic Processes, $4^{\text {th }}$ edition, International Edition, Mc-Graw-Hill.

[49]. Petrone, G., Axerio-Cilies, J., Quagliarella, D., Iaccarino, G. "A probabilistic nondominated sorting ga for optimization under uncertainty," Engineering Computations (Swansea, Wales), Vol. 30, No. 8, pp. 1054-1085, 2013.

[50]. Rocco, S.C.M., "Effects of the transition rate uncertainty on the steady state probabilities of Markov models using interval arithmetic", Proceedings of the Institution of Mechanical Engineers, Part O: Journal of Risk and Reliability Vol. 226, No. 1, pp. 234-245, 2011. 
[51]. Rockafellar, R.T., Uryasev, S., "Conditional value-at-risk for general loss distributions", Journal of Banking and Finance, Vol 26, No. 14, pp. 43-71, 2002

[52]. Sanchez, A., Carlos, S., Martorell, S., Villanueva, J.F. "Addressing imperfect maintenance modelling uncertainty in unavailability and cost based optimization," Reliability Engineering \& System Safety, Vol. 94, No. 1, pp. 22-32, 2009.

[53]. Sarykalin, S., Fraud, G., Serraino, G., Uryasev, S., "Value-at-Risk vs. Conditional Value-at-Risk in Risk Management and Optimization", Tutorials in Operations Research, INFORMS, 2008.

[54]. Shaked M., Shanthikumar, J. G. "Stochastic Orders and their Applications," Associated Press, 1994.

[55]. ŠKkulj, D., "Discrete time Markov chains with interval probabilities", International Journal of Approximate Reasoning, Vol. 50,pp. 1314-1329, 2009.

[56]. Trebi-Ollennu, A., White, B.A., "Multiobjective fuzzy genetic algorithm optimisation approach to nonlinear control system design", IEE Proceedings - Control Theory and Applications Vol. 144, pp. 137-142, 1997.

[57]. Villanueva, J.F., Sanchez, A.I., Carlos, S., Martorell, S. "Genetic algorithm-based optimization of testing and maintenance under uncertain unavailability and cost estimation: A survey of strategies for harmonizing evolution and accuracy," Reliability Engineering \& System Safety, Vol. 93, No. 12, pp. 1830-1841, 2008.

[58]. Whitmore G., Third-degree stochastic dominance, "American Economic Review," Vol. 60, No. 3, pp. 457-459, 1970.

[59]. Zio E., Baraldi P., Pedroni N., "Optimal power system generation scheduling by multiobjective genetic algorithms with preferences", Reliability Engineering and System Safety, Vol. 94, pp. 432- 444, 2009.

[60]. Zio, E., Bazzo, R., "A clustering procedure for reducing the number of representative solutions in the Pareto Front of multiobjective optimization problems," European Journal of Operational Research, Vol. 210, No. 3, pp. 624-634, 2011.

[61]. Zio, E. "The Monte Carlo Simulation Method for System Reliability and Risk Analysis", Springer, 2013

[62]. Zio, E., Compare, M., "A snapshot on maintenance modeling and applications", Marine Technology and Engineering, Vol. 2, pp. 1413-1425, 2013.

[63]. Zio, E., Compare, M., "Evaluating maintenance policies by quantitative modeling and analysis", Reliability Engineering and System Safety, Vol. 109, pp. 53-65, 2013. 